\title{
Attachment of Human C5a des Arg to Its Cochemotaxin Is Required for Maximum Expression of Chemotactic Activity
}

\author{
H. Daniel Perez ${ }^{\star}$, Dennis E. Chenoweth‡, and Ira M. Goldstein*
}

${ }^{*}$ Rosalind Russell Arthritis Research Laboratory, Department of Medicine, University of California, San Francisco, San Francisco, California 94143-0868; and $\ddagger$ Department of Pathology, Veterans Administration Medical Center, University of California,

San Diego, La Jolla, California 92093

\begin{abstract}
The chemotactic activity of human C5a des Arg is enhanced significantly by an anionic polypeptide (cochemotaxin) in normal human serum and plasma. We have found that the cochemotaxin attaches to the oligosaccharide chain of native C5a des Arg to form a complex with potent chemotactic activity for human polymorphonuclear leukocytes. Although capable of enhancing the chemotactic activity of native C5a des Arg, the cochemotaxin had no effect on the chemotactic activity of either deglycosylated C5a des Arg, native C5a, or $\boldsymbol{N}$-formyl-methionyl-leucyl-phenylalanine. Of the known components of the oligosaccharide chain, only sialic acid prevented enhancement by the cochemotaxin of the chemotactic activity exhibited by native C5a des Arg. Sialic acid also prevented the formation of $\mathrm{C5a}$ des Arg-cochemotaxin complexes, detected by acid polyacrylamide gel electrophoresis, molecular sieve chromatography on polyacrylamide gels, and sucrose density gradient ultracentrifugation.
\end{abstract}

\section{Introduction}

Activation of the complement system in human serum results in the generation of chemotactic activity for human polymorphonuclear leukocytes $(\mathrm{PMN})^{1}(1)$. This activity is attributable primarily to the complement C5-derived peptides, C5a and C5a des Arg (1-5). Highly purified human C5a exhibits anaphylatoxin activity (6) and is a potent chemotactic factor for PMN (2-5). In human serum, however, $\mathrm{C} 5 \mathrm{a}$ is converted rapidly by the naturally occurring enzyme, carboxypeptidase $\mathrm{N}$, to the less active, but more stable peptide, C5a des Arg $(6,7)$. Highly purified human C5a des Arg almost completely lacks anaphylatoxin activity (6) and is 10-20-fold less potent than C5a as a chemoattractant for PMN (2-5). We reported previously that nonchemotactic concentrations of human C5a des Arg become chemotactically active, however, when assayed in the presence of a naturally occurring anionic polypeptide, the $\mathrm{C} 5 \mathrm{a}$ des Arg cochemotaxin $(4,5)$. The profile of chemotactic activity exhibited by mixtures of C5a des Arg and its cochemotaxin very closely re-

This paper was presented at the annual meeting of the American Federation for Clinical Research, May 5 1985, and published in abstract form (1985, Clin. Res., 33:384A [Abstr.]). 1986.

Received for publication 11 March 1986 and in revised form 14 July

1. Abbreviations used in this paper: $\mathrm{C} 5 \mathrm{a}-\mathrm{CHO}$, deglycosylated $\mathrm{C} 5 \mathrm{a} ; \mathrm{C5a}$ des Arg-CHO, deglycosylated C5a des Arg; FMLP, $N$-formyl-methionylleucyl-phenylalanine; PAGE, polyacrylamide gel electrophoresis; PMN, polymorphonuclear leukocyte(s); ZTS, zymosan-treated serum.

The Journal of Clinical Investigation, Inc.

Volume 78, December 1986, 1589-1595 sembles that exhibited by either dilutions of activated serum or highly purified C5a. Thus, C5a des Arg and its cochemotaxin probably account for most of the chemotactic activity that is generated in human serum after limited complement activation.

The mechanism by which the cochemotaxin augments the chemotactic activity of C5a des Arg is unknown. It is possible, however, that the anionic cochemotaxin binds to C5a des Arg (a cationic peptide) to form a "complex" with potent chemotactic activity. Indirect evidence of such a complex was provided by Beebe et al. (8), who isolated from zymosan-activated human serum an anionic, C5-derived chemotactic factor with an apparent molecular weight of 65,000 . Treatment of this factor with reducing agents resulted in loss of chemotactic activity. Additional evidence that C5a des Arg and its cochemotaxin might form a physical complex was provided by Kreutzer et al. (9), who demonstrated by basic polyacrylamide gel electrophoresis (PAGE) a previously unrecognized anionic "fragment" of C5 that was formed in whole human serum as a consequence of complement activation.

A mechanism was described recently whereby the biologic activities of human C5-derived peptides can be modulated. Gerard and Hugli (10) found that when the single oligosaccharide chain of C5a des Arg was removed by glycosidases, leaving a single glucosamine residue attached to asparagine-64, the peptide regained anaphylatoxin activity. Deglycosylated C5a des Arg (C5a des Arg-CHO) contracted the guinea pig ileum and enhanced vascular permeability in guinea pig skin. Gerard et al. (11) subsequently demonstrated that human $\mathrm{C} 5 \mathrm{a}$ des Arg- $\mathrm{CHO}$ was significantly more active than native $\mathrm{C} 5 \mathrm{a}$ des Arg with respect to its ability to promote PMN chemotaxis. Native C5a and deglycosylated $\mathrm{C5a}$ (C5a-CHO), on the other hand, behaved identically. These findings suggest that the oligosaccharide moiety does not influence the biologic activities of human C5a, but acts as a negative modulator of the biologic activities of C5a des Arg.

In this report, studies are described in which we examined effects of the cochemotaxin on the chemotactic activities of native C5a des Arg and C5a des Arg-CHO. We have confirmed that $\mathrm{C} 5 \mathrm{a}$ des $\mathrm{Arg}-\mathrm{CHO}$ is significantly more potent than native $\mathrm{C5a}$ des Arg as a chemoattractant for PMN, and present evidence that the cochemotaxin does, in fact, form a physical complex with native $\mathrm{C5a}$ des Arg, possibly by attaching to a sialic acid residue on the oligosaccharide portion of the molecule.

\section{Methods}

Preparation of C5-derived peptides and the cochemotaxin. C5a was isolated from yeast-activated human serum containing the carboxypeptidase $\mathrm{N}$ inhibitor, 2-mercaptomethyl-3-guanidinoethylthiopropanoic acid (Calbiochem-Behring Corp., La Jolla, CA), using a minor modification of the method described by Hugli et al. (12). Final purification of C5a was accomplished by immunoabsorption chromatography on a column of Sepharose 4B (Pharmacia Fine Chemicals, Piscataway, NJ) to which 
a monoclonal antibody raised against human C5a was linked convalently. $\mathrm{C} 5 \mathrm{a}$ was deglycosylated enzymatically and $\mathrm{C5a}-\mathrm{CHO}$ was purified by gel filtration as described by Gerard and Hugli (10). Amino sugar analysis revealed that $\mathrm{C} 5 \mathrm{a}-\mathrm{CHO}$ contained $\sim 1 \mathrm{~mol}$ of glucosamine linked to asparagine-64 per mole of peptide (13). The polypeptide chain of C5a$\mathrm{CHO}$ was judged to be intact by criteria described previously $(10,11)$. C5a des Arg and C5a des Arg-CHO were prepared by treating the respective C5a preparations $(15 \mu \mathrm{g} / \mathrm{ml})$ with insoluble carboxypeptidase B (Worthington Biochemical Corp., Freehold, NJ), as described previously $(3,5)$. Based on assays for anaphylatoxin activity, residual native C5a, if any, could not account for $>0.04 \%$ of the total protein in the C5a des Arg preparations $(3,5)$. Porcine C5a was generously provided by Dr. Tony Hugli (Scripps Clinic and Research Foundation, La Jolla, CA). Porcine $\mathrm{C} 5 \mathrm{a}$ des Arg was prepared by treating porcine $\mathrm{C} 5 \mathrm{a}$ with insoluble carboxypeptidase $\mathrm{B}$.

Human C5a was radiolabeled with $\mathrm{Na}^{125}$ I (Amersham Corp., Arlington Heights, IL) by the solid-phase lactoperoxidase-glucose oxidase method (Enzymobead; Bio-Rad Laboratories, Richmond, CA), as described previously $(14,15)$. Free $\mathrm{Na}^{125}$ I and "altered" radiolabeled C5a were separated from antigenically reactive ${ }^{125} \mathrm{I}-\mathrm{C} 5 \mathrm{a}$ by immunoabsorption chromatography on a column of rabbit anti-C5a IgG-Sepharose 4B. Native ${ }^{125} \mathrm{I}-\mathrm{C} 5 \mathrm{a}$ was eluted with $0.2 \mathrm{M}$ glycine- $\mathrm{HCl}$ (pH 2.3) and immediately neutralized with $1.0 \mathrm{M} \mathrm{K}_{2} \mathrm{HPO}_{4}$. The specific activity of ${ }^{125} \mathrm{I}$ C5a was $\sim 2.6 \times 10^{6} \mathrm{cpm} / \mu \mathrm{g}$ protein. Radiolabeled C5a was judged to have retained its native character because $>90 \%$ was bound by an excess of rabbit anti-human $\mathrm{C5a}$ Ig in a solid-phase radioimmunoassay (15) and was $>85 \%$ bound by PMN (16). Furthermore, labeled and unlabeled C5a were equally active with respect to their ability to induce PMN chemotaxis. Radiolabeled C5a des Arg was prepared by incubating ${ }^{125} \mathrm{I}$ C5a with insoluble carboxypeptidase $\mathrm{B}(3,5)$.

The C5a des Arg cochemotaxin was prepared as described previously (5). Briefly, heat-inactivated $\left(56^{\circ} \mathrm{C}\right.$ for $\left.30 \mathrm{~min}\right)$ serum was subjected to stepwise fractionation with saturated ammonium sulfate (35 and $70 \%$, $\mathrm{vol} / \mathrm{vol}$ ), and the resulting $70 \%$ pellet subjected to chromatography on Sephadex G-75 (Pharmacia Fine Chemicals). Active fractions were pooled and chromatographed on a $1.5 \times 30 \mathrm{~cm}$ column of DEAE-Sephacel (Pharmacia Fine Chemicals). Final purification was accomplished by high performance liquid chromatography on a Spherogel SW-3000 protein analysis column (Beckman Instruments, Inc., Palo Alto, CA).

The purified cochemotaxin was radioiodinated by the solid-phase lactoperoxidase-glucose oxidase method (14), and was separated from free $\mathrm{Na}^{125} \mathrm{I}$ by elution from a $1.0 \times 30 \mathrm{~cm}$ column of Sephadex G-10 (Pharmacia Fine Chemicals) with phosphate $(10 \mathrm{mM})$-buffered $140 \mathrm{mM}$ $\mathrm{NaCl}$ (pH 7.4). The specific activity of the cochemotaxin was $\sim 4.7 \times 10^{5}$ $\mathrm{cpm} / \mu \mathrm{g}$ protein. As was the case with $\mathrm{C} 5 \mathrm{a}$, radioiodination did not alter the biologic activity of the cochemotaxin. For some experiments, purified cochemotaxin $(1.0 \mu \mathrm{g})$ and purified native C5a des Arg $(10 \mu \mathrm{g})$ were treated with $2 \mathrm{U}$ of solid-phase neuraminidase from Clostridium perfringens (Sigma Chemical Co., St. Louis, MO) in phosphate (10 mM)buffered $140 \mathrm{mM} \mathrm{NaCl}$ (pH 6.5) for $30 \mathrm{~min}$ at room temperature (17).

C5-derived chemotactic activity was generated in fresh human serum by adding zymosan $(1.0 \mathrm{mg} / \mathrm{ml})$ (ICN Nutritional Biochemicals Div., International Chemical and Nuclear Corp., Cleveland, $\mathrm{OH})(4,5)$. After 15 min of incubation at $37^{\circ} \mathrm{C}$, the zymosan-serum suspension was centrifuged at $3000 \mathrm{~g}$ for $10 \mathrm{~min}$, and the particle-free supernatant used as a source of C5-derived chemotactic peptides.

Preparation of leukocyte suspensions. Leukocyte suspensions containing 97-99\% PMN were prepared from heparinized (10 U/ml) venous blood by centrifugation on Hypaque/Ficoll gradients, dextran sedimentation, and hypotonic lysis of erythrocytes (18). Cells were washed once with $140 \mathrm{mM} \mathrm{NaCl}$, and finally suspended in phosphate $(10 \mathrm{mM})$-buffered $140 \mathrm{mM} \mathrm{NaCl}$ (pH 7.4) supplemented with $0.6 \mathrm{mM} \mathrm{CaCl}_{2}, 1.0 \mathrm{mM}$ $\mathrm{MgCl}_{2}$ and $20 \mathrm{mg} / \mathrm{ml}$ bovine serum albumin (Gibco, Grand Island, NY).

$P M N$ chemotaxis. Directed migration (chemotaxis) of PMN was measured using a minor modification (19) of the leading front method of Zigmond and Hirsch (20). Stimulated random motility in response to albumin-containing buffer (21) and total migration in response to chemoattractants are reported as the distance $(\mu \mathrm{m} / 35 \mathrm{~min})$ that the lead- ing front of cells migrated into $3.0-\mu \mathrm{m}$ pore-diameter cellulose nitrate filters (Sartorius Filters, Inc., Hayward, CA) separating the upper, or cell compartments, from the lower, or stimulus compartments, of modified Boyden chambers (Nucleopore Corp., Pleasanton, CA). Chemotaxis (net migration) was calculated by subtracting stimulated random motility from total migration. Duplicate chambers were used in each experiment and 10 fields were examined in each filter.

In some experiments, the method described by Maderazo and Woronick (22) was used to assess the migratory behavior of the entire population of PMN. Leukotactic indices, which reflect the mean distance travelled by all cells, were determined by counting the number of cells on the surfaces of the micropore filters and at various depths $(10-\mu \mathrm{m}$ intervals) in the filters using an image analyzer (Optomax Inc., Hollis, $\mathrm{NJ}$ ) interfaced with an Apple II computer (Apple Computer, Inc., $\mathrm{Cu}$ pertino, CA).

$P A G E$. Electrophoresis was performed in $7.5 \%$ polyacrylamide tube gels using a buffer containing $0.188 \mathrm{M}$ glycine and $0.188 \mathrm{M}$ acetic acid (pH 3.6). Gels were run at $3 \mathrm{~mA} /$ tube for $2 \mathrm{~h}$, after which they were removed and sliced in 2-mm sections.

Sucrose density gradient centrifugation. Peptides were diluted in 1.0 $\mathrm{ml}$ of $10 \mathrm{mM}$ Hepes buffer (pH 7.4) containing $1.0 \mathrm{mM}$ EDTA (Sigma Chemical Co.), and layered onto $12 \mathrm{ml}$ of a linear gradient of sucrose $\left(2.5-20 \%\right.$, wt/vol) in polyallomer tubes $\left(9 / 16^{\prime \prime}\right.$; Beckman Instruments, Inc.) (23). Layered samples were centrifuged in a rotor (model Ti 40; Beckman Instruments, Inc.) at $180,000 \mathrm{~g}$ for $18 \mathrm{~h}$ at $4^{\circ} \mathrm{C}$. Fractions $(0.5$ $\mathrm{ml}$ ) were collected by puncturing the bottom of each tube using a fractionator (Beckman Instruments, Inc.). Egg white lysozyme (Sigma Chemical Co.), fatty-acid-free human albumin (Sigma Chemical Co.) and purified human $\operatorname{IgG~} \mathrm{F}\left(\mathrm{ab}^{\prime}\right)_{2}$ fragments were used as standards. The migration of standard proteins (alone and in combination) was determined by measuring ultraviolet absorbance at $280 \mathrm{~nm}$.

Other materials. Bio-Gel P-150 was from Bio-Rad Laboratories. $N$ acetyl-d-glucosamine, mannose, galactose, and sialic acid were from Sigma Chemical Co. The synthetic chemotactic peptide, $N$-formylmethionyl-leucyl-phenylalanine (FMLP), was from Peninsula Laboratories, Inc. (Belmont, CA).

\section{Results}

Chemotactic activities of C5a, C5a des Arg, and C5a des Arg$\mathrm{CHO}$. Highly purified human $\mathrm{C} 5 \mathrm{a}$, at a concentration of $1.0 \mathrm{ng} /$ ml, significantly enhanced directed migration of human PMN (Table I). In contrast, C5a des Arg did not exhibit chemotactic

Table I. Chemotactic Activities of C5a, Native C5a des Arg, and C5a des Arg-CHO

\begin{tabular}{lcc}
\hline Stimulus & & PMN Migration* \\
\hline & $n g / m l$ & $\mu m / 35 \mathrm{~min}$ \\
Buffer & & $84.7 \pm 2.2$ \\
C5a & 1.0 & $104.6 \pm 2.0 \ddagger$ \\
Native C5a des Arg & 1.0 & $84.1 \pm 1.6$ \\
& 5.0 & $87.4 \pm 2.7$ \\
& 10 & $92.6 \pm 1.8 \S$ \\
C5a des Arg-CHO & 20 & $105.6 \pm 4.1 \ddagger$ \\
& 0.5 & $83.0 \pm 2.3$ \\
& 1.0 & $92.7 \pm 2.6 \S$ \\
& 5.0 & $102.4 \pm 2.5 \ddagger$ \\
& 10 & $112.1 \pm 2.0 \ddagger$ \\
& 20 & $122.0 \pm 2.6 \ddagger$
\end{tabular}

\footnotetext{
* Results represent mean values ( $\pm \mathrm{SE}$ ) obtained in three experiments using PMN from different donors. $\ddagger P$ vs. buffer alone $<0.01$ ( $t$ test). $\S P$ vs. buffer alone $<0.05$ ( $t$ test).
} 
activity at concentrations $<10 \mathrm{ng} / \mathrm{ml}$ (Table I). At a concentration of $20 \mathrm{ng} / \mathrm{ml}, \mathrm{C} 5 \mathrm{a}$ des Arg was as potent as $1.0 \mathrm{ng} / \mathrm{ml} \mathrm{C5a}$. C5a des Arg-CHO, on the other hand, exhibited significant chemotactic activity at a concentration of $1.0 \mathrm{ng} / \mathrm{ml}$, and at a concentration of $5.0 \mathrm{ng} / \mathrm{ml}$, exhibited the same chemotactic potency as $20 \mathrm{ng} / \mathrm{ml} \mathrm{C5a}$ des Arg and $1.0 \mathrm{ng} / \mathrm{ml} \mathrm{C5a} \mathrm{(Table} \mathrm{I).} \mathrm{thus,}$ based on results using the leading front assay. C5a des Arg-CHO appeared to be fourfold more active than native C5a des Arg, but fivefold less active than $\mathrm{C} 5 \mathrm{a}$. Virtually identical results were obtained when chemotaxis was measured by the method of Maderazo and Woronick (22). Leukotactic indices for buffer alone, native C5a des Arg (5.0 and $20 \mathrm{ng} / \mathrm{ml}$ ), and C5a des ArgCHO $(5.0$ and $20 \mathrm{ng} / \mathrm{ml})$ were 8.0, 8.2, 14.2, 13.1, and 20.6, respectively (average of two experiments).

The chemotactic activity of native C5 des Arg was enhanced significantly, and in a concentration-dependent fashion, by the purified cochemotaxin (Fig. 1). Whereas $5.0 \mathrm{ng} / \mathrm{ml} \mathrm{C5a}$ des Arg alone did not stimulate directed migration of PMN, the same concentration of $\mathrm{C} 5 \mathrm{a}$ des Arg exhibited significant chemotactic activity in the presence of the cochemotaxin. The cochemotaxin by itself was not chemotactic, nor did it enhance the chemotactic activity exhibited by $0.5 \mathrm{ng} / \mathrm{ml} \mathrm{C5a}$ des Arg-CHO (Fig. 1). Similarly, the cochemotaxin did not influence the chemotactic activity exhibited by either $5.0 \mathrm{ng} / \mathrm{ml} \mathrm{C5a}$ des Arg-CHO or suboptimal concentrations of C5a and FMLP (data not shown).

Interactions between the cochemotaxin and C5a des Arg. To determine whether the cochemotaxin interacts with components of the oligosaccharide chain of C5a des Arg, the chemotactic activity expressed by mixtures of the two polypeptides was measured in the presence and absence of various monosaccharides. Of the monosaccharides that are known to be components of the oligosaccharide chain (13), only sialic acid proved to be capable of preventing enhancement by the cochemotaxin of the chemotactic activity exhibited by $5.0 \mathrm{ng} / \mathrm{ml} \mathrm{C5a}$ des Arg (Table II). In contrast, sialic acid did not influence directed migration of PMN toward chemotactic concentrations of either C5a (1.0 $\mathrm{ng} / \mathrm{ml})$, C5a des Arg $(20 \mathrm{ng} / \mathrm{ml})$, or FMLP $\left(10^{-8} \mathrm{M}\right)$ (Table III). To determine whether the negative charge of sialic acid contributed to its effects, experiments were performed with the polyanion heparin. Heparin (up to $5.0 \mathrm{U} / \mathrm{ml}$ ), however, failed to influence the chemotactic activity exhibited by mixtures of native C5a des Arg and cochemotaxin (Table III).

Evidence was found in two ways that the sialic acid residue involved in the C5a des Arg-cochemotaxin interaction most likely is located on C5a des Arg. First, treatment of the cochemotaxin with neuraminidase had no effect on its ability to en-

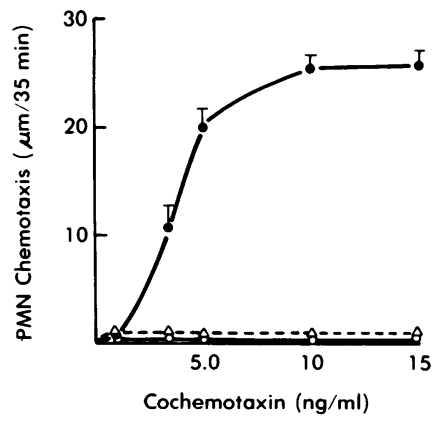

Figure 1. Effects of purified cochemotaxin on the chemotactic activities of native $\mathrm{C} 5 \mathrm{a}$ des Arg $(5.0 \mathrm{ng} / \mathrm{ml})$ (solid circle) and deglycosylated C5a des $\operatorname{Arg}(0.5 \mathrm{ng} / \mathrm{ml})$ (open circle). The cochemotaxin alone (open triangle) did not exhibit chemotactic activity. Results represent net PMN migration (total migration minus migration in response to buffer alone), and are mean values $( \pm S E)$ from three experiments using PMN from different donors.
Table II. Effects of Various Sugars on the Chemotactic Activity of Native C5a des Arg plus the Cochemotaxin

\begin{tabular}{lc}
\hline Stimulus & PMN migration* \\
\hline & $\mu m / 35$ min \\
Buffer & $85.1 \pm 1.5$ \\
C5a des Arg $(5.0 \mathrm{ng} / \mathrm{ml})$ & $86.9 \pm 2.2$ \\
C5a des Arg $(5.0 \mathrm{ng} / \mathrm{ml})$ & \\
$\quad+$ cochemotaxin $(10 \mathrm{ng} / \mathrm{ml})$ & $109.7 \pm 3.5 \ddagger$ \\
+ N-acetyl-D-glucosamine $(5.0 \mathrm{mM})$ & $108.6 \pm 4.7 \ddagger$ \\
+ galactose $(5.0 \mathrm{mM})$ & $108.7 \pm 4.4 \ddagger$ \\
+ mannose $(5.0 \mathrm{mM})$ & $108.0 \pm 2.9 \ddagger$ \\
+ sialic acid $(5.0 \mathrm{mM})$ & $88.8 \pm 2.1$ \\
& \\
\hline
\end{tabular}

* Results represent mean values $( \pm \mathrm{SE})$ obtained in three experiments using PMN from different donors.

$\ddagger P$ vs. buffer alone $<0.01$ ( $t$ test).

hance the chemotactic activity of native C5a des Arg (Table III). Second, treatment with neuraminidase enhanced the chemotactic activity of native C5a des Arg. Whereas $5.0 \mathrm{ng} / \mathrm{ml}$ native C5a des Arg was devoid of chemotactic activity, $5.0 \mathrm{ng} / \mathrm{ml} \mathrm{neur-}$ aminidase-treated C5a des Arg caused net PMN migration (26.5 $\mu \mathrm{m} / 35 \mathrm{~min}, n=2)$. Furthermore, the cochemotaxin $(10 \mathrm{ng} / \mathrm{ml})$ did not affect the chemotactic activity of neuraminidase-treated C5a des Arg (1.0-5.0 ng/ml) (not shown).

Effects of sialic acid on the chemotactic activity exhibited by mixtures of C5a des Arg and the cochemotaxin were concentration-dependent (Fig. 2). Significant inhibition was observed with $2.0 \mathrm{mM}$ sialic acid $(P<0.01, t$ test), while complete inhibition occurred at concentrations between 3.0 and $5.0 \mathrm{mM}$.

To determine whether there was a true physical interaction between the two polypeptides, highly purified ${ }^{125} \mathrm{I}-\mathrm{C} 5 \mathrm{a}$ des Arg was subjected to $7.5 \%$ PAGE under acidic conditions in the

Table III. Effects of Sialic Acid, Heparin, and Neuraminidasetreated Cochemotaxin on the Chemotactic Activities of C5a, C5a des Arg, and FMLP

\begin{tabular}{cc}
\hline Stimulus & $\begin{array}{l}\text { Net PMN } \\
\text { migration }\end{array}$ \\
\hline & $\mu m / 35$ min \\
C5a $(1.0 \mathrm{ng} / \mathrm{ml})$ & $37.1 \pm 1.7$ \\
+ sialic acid $(5.0 \mathrm{mM})$ & $37.4 \pm 2.3$ \\
C5a des Arg $(20 \mathrm{ng} / \mathrm{ml})$ & $35.8 \pm 2.2$ \\
+ sialic acid $(5.0 \mathrm{mM})$ & $37.2 \pm 2.7$ \\
FMLP $\left(10^{-8} \mathrm{M}\right)$ & $48.3 \pm 2.4$ \\
+ sialic acid $(5.0 \mathrm{mM})$ & $48.5 \pm 1.9$ \\
C5a des Arg $(5.0 \mathrm{ng} / \mathrm{ml})$ & \\
$\quad+$ cochemotaxin $(10 \mathrm{ng} / \mathrm{ml})$ & $36.0 \pm 1.6$ \\
+ heparin $(5 \mathrm{U} / \mathrm{ml})$ & $36.1 \pm 2.7$ \\
C5a des Arg $(5.0 \mathrm{ng} / \mathrm{ml})$ & \\
$\quad+$ neuraminidase-treated & \\
cochemotaxin $(10 \mathrm{ng} / \mathrm{ml})$ & $33.9 \pm 2.6$ \\
\hline
\end{tabular}

* Net PMN migration was calculated by subtracting stimulated random motility from total migration. Results represent mean values $( \pm \mathrm{SE})$ obtained in three experiments. 


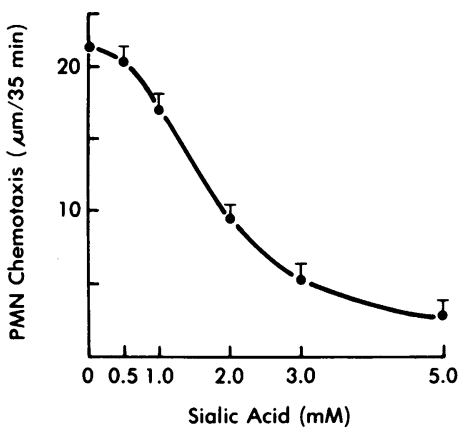

Figure 2. Effects of sialic acid on the chemotactic activity of native C5a des Arg $(5.0 \mathrm{ng} / \mathrm{ml})$ plus the coche$\operatorname{motaxin}(10 \mathrm{ng} / \mathrm{ml})$ (mean \pm SE, $n=3$ ). Results represent net PMN migration (i.e., total migration minus migration in response to buffer alone).

presence and absence of cochemotaxin. As reported previously (3), ${ }^{125} \mathrm{I}-\mathrm{C} 5 \mathrm{a}$ des Arg migrated readily into acid gels (Fig. $3 \mathrm{~A}$ ). Radioactivity was detected in a sharp, symmetric peak, which indicated the absence of contaminating proteins. Migration of ${ }^{125} \mathrm{I}$-C5a des Arg into acid gels was significantly retarded, however, when it was electrophoresed in the presence of cochemotaxin (Fig. $3 \mathrm{~B}$ ). This effect was reversed by adding $5.0 \mathrm{mM}$ sialic acid to the mixture of ${ }^{125} \mathrm{I}-\mathrm{C} 5 \mathrm{a}$ des Arg plus cochemotaxin (Fig. $3 \mathrm{~B}$ ). Sialic acid (5.0 mM) had no effect on the migration of ${ }^{125} \mathrm{I}-\mathrm{C} 5 \mathrm{a}$ des Arg alone (not shown).

To confirm the results obtained using acid PAGE, molecular sieve chromatography was performed on Bio-Gel P-150. $\left[{ }^{125} \mathrm{I}\right]-$ C5a des Arg was applied to a $1.0 \times 20-\mathrm{cm}$ column of the polyacrylamide gel and eluted with Hanks' balanced salt solution (pH 7.4). Fractions $(0.4 \mathrm{ml})$ were collected and radioactivity was measured by counting gamma emissions. Radiolabeled C5a des Arg eluted as a symmetric peak with an apparent molecular weight of $\sim 11,000$ (Fig. $4 \mathrm{~A}$ ). When a mixture of radiolabeled C5a des Arg and cochemotaxin was chromatographed under identical conditions, the peak of radioactivity eluted with an apparent molecular weight of 85,000 (Fig. $4 \mathrm{~A}$ ). Again, this effect of the cochemotaxin was prevented by $5.0 \mathrm{mM}$ sialic acid (not shown).

Identical experiments were performed using either unlabeled native $\mathrm{C} 5 \mathrm{a}$ des Arg or unlabeled $\mathrm{C5a}$ des Arg-CHO and radiolabeled cochemotaxin. Chromatography of purified ${ }^{125} \mathrm{I}$-coche-

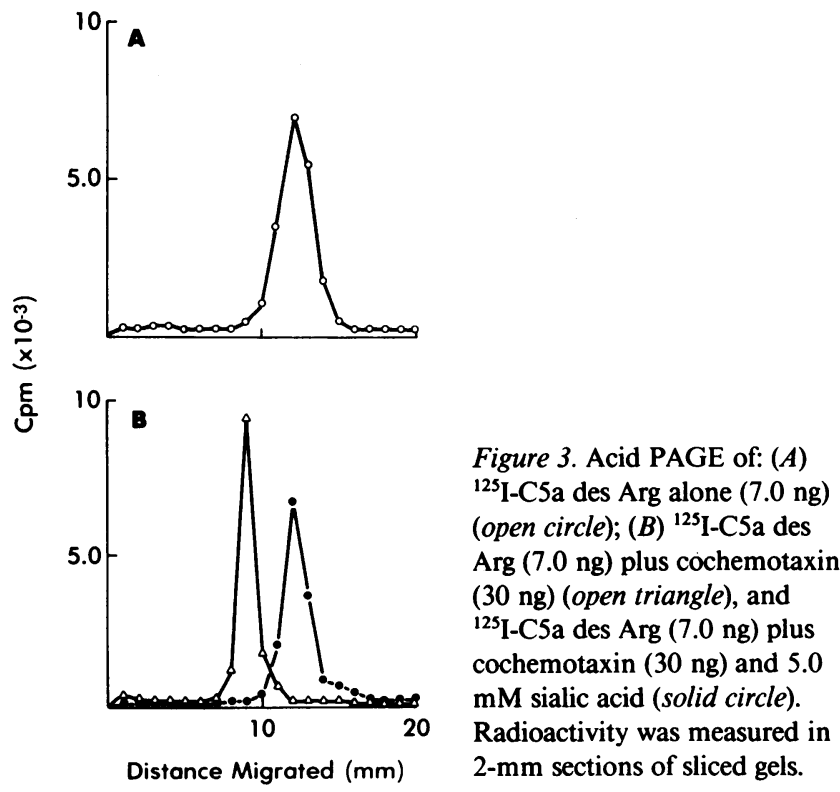

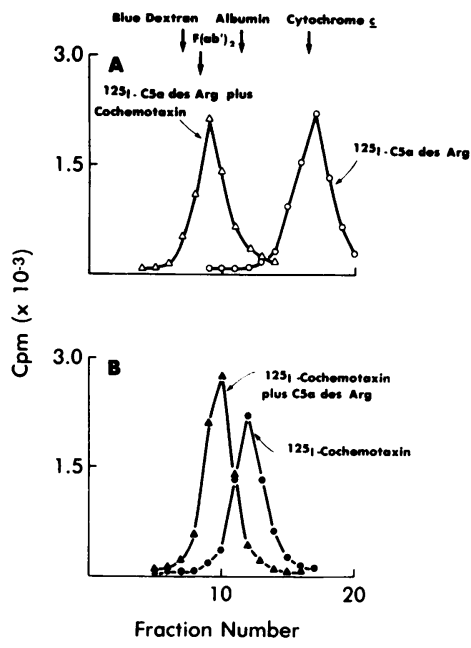

Figure 4. Elution profiles of radioactivity after molecular sieve chromatography of: $(A){ }^{125} \mathrm{I}-\mathrm{C} 5 \mathrm{a}$ des $\operatorname{Arg}(5.0$ $\mathrm{ng}$ ) with (open triangle) and without (open circle) cochemotaxin (10 $\mathrm{ng}$ ); and $(B)$ ${ }^{125} \mathrm{I}$-cochemotaxin (10 ng) with (solid triangle) and without (solid circle) unlabeled C5a des Arg (5.0 ng). Chromatography was performed on a $1.0 \times 20$-cm column of Bio-Gel P-150, and radioactivity measured in 0.4-ml fractions eluted with Hanks' balanced salt solution.

motaxin alone yielded a symmetric peak of radioactivity that eluted with an apparent molecular weight of $\sim 60,000$ (Fig. 4 $B)$. As expected, none of the fractions from this peak contained chemotactic activity for PMN (not shown). Identical results were obtained when ${ }^{125} \mathrm{I}$-cochemotaxin was chromatographed in the presence of $\mathrm{C} 5 \mathrm{a}$ des Arg-CHO. However, when a mixture of ${ }^{125} \mathrm{I}$ cochemotaxin plus native $\mathrm{C5a}$ des Arg was chromatographed under identical conditions, the peak of radioactivity eluted with an apparent molecular weight of $\sim 85,000$ (Fig. $4 \mathrm{~B}$ ). The fraction from this peak that contained the greatest amount of radioactivity also contained significant chemotactic activity for PMN $(95.1 \pm 2.0 \mu \mathrm{m} / 35 \mathrm{~min}$ vs. $80.4 \pm 2.1 \mu \mathrm{m} / 35 \mathrm{~min}$ for buffer alone). Identical results were obtained when mixtures of ${ }^{125} \mathrm{I}$-cochemotaxin and C5a des Arg were chromatographed using both low $(50 \mathrm{mM} \mathrm{NaCl})$ and high $(200 \mathrm{mM} \mathrm{NaCl})$ ionic strength buffers (not shown).

That native C5a des Arg forms a physical complex with the cochemotaxin was confirmed further by sucrose density gradient ultracentrifugation. As Fig. 5 shows, native ${ }^{125} \mathrm{I}-\mathrm{C} 5 \mathrm{a}$ des Arg alone migrated into sucrose gradients with an apparent molecular weight of $14,200 \pm 1,600$. When a mixture of ${ }^{125}$ I-C 5a des Arg and cochemotaxin was analyzed under identical conditions, a peak of radioactivity was detected with an apparent molecular weight of $85,300 \pm 3,000$. Identical results were obtained when ${ }^{125} \mathrm{I}$-C5a des Arg was mixed with neuraminidase-treated cochemotaxin.

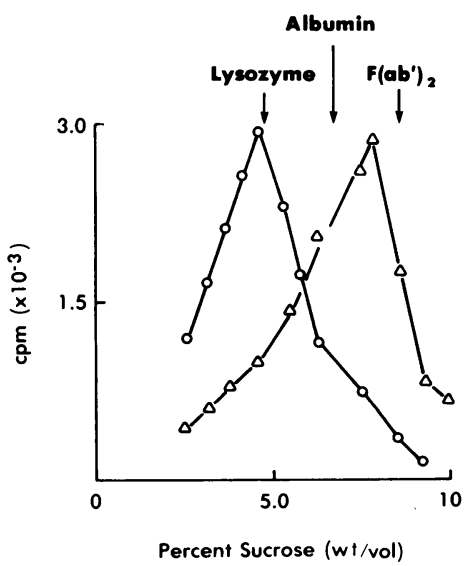

Figure 5. Analysis of the interaction between $\mathrm{C5}$ a des Arg and cochemotaxin by sucrose density gradient ultracentrifugation. ${ }^{125} \mathrm{I}-\mathrm{C} 5 \mathrm{a}$ des Arg (5.0 ng) alone (open circle); ${ }^{125} \mathrm{I}-\mathrm{C} 5 \mathrm{a}$ des $\mathrm{Arg}(5.0$ ng) plus cochemotaxin ( 10 ng) (open triangle) 
Experiments were performed using molecular sieve chromatography to determine whether zymosan-treated human serum (ZTS) contains high molecular weight, C5-derived chemotactic activity (i.e., complexes of C5a des Arg and cochemotaxin). We $(4,5,19)$, and others $(24,25)$, have demonstrated previously that the bulk of $\mathrm{C5}$-derived chemotactic activity elutes after chromatography of ZTS on Sephadex G-75 in a single peak with an apparent molecular weight of $\sim 15-20,000$. We have concluded that fractions in this peak contain a mixture of C5a des Arg and cochemotaxin, and have postulated that the cochemotaxin elutes with a lower apparent molecular weight than expected because it binds to Sephadex $(4,5)$. ZTS $(0.2 \mathrm{ml})$ was applied to a $1.0 \times 20-\mathrm{cm}$ column of Bio-Gel P-150 and was eluted with Hanks' balanced salt solution. Fractions $(0.4 \mathrm{ml})$ were collected and assayed for the presence of chemotactic activity. As Fig. 6 shows when fractions were assayed at a concentration of $2.5 \%$ chemotactic activity was detected in a single peak which eluted with an apparent molecular weight of 85,000 . Chemotactic activity in these fractions resisted heating at $56^{\circ} \mathrm{C}$ for $30 \mathrm{~min}$, but was abolished by treatment with antibodies to human C5 (not shown). It should be noted, however, that when column fractions were tested at a concentration of $25 \%, \mathrm{C5}$ derived chemotactic activity (presumably C5a des Arg alone) also was detected in a peak eluting with an estimated molecular weight of 13,000 (not shown).

Effects of human cochemotaxin, porcine serum, and rabbit serum on the chemotactic activities of porcine C5a, porcine C5a des Arg, and human C5a des Arg. It has been reported previously that porcine $\mathrm{C} 5 \mathrm{a}$ and $\mathrm{C5a}$ des Arg are chemotactic for human PMN (11). In contrast to human C5a, however, porcine C5a is devoid of carbohydrate (11). Thus, it was of interest to determine the effect of the cochemotaxin on the chemotactic activities of suboptimal concentrations of porcine C5a and C5a des Arg. As Table IV shows, the purified cochemotaxin enhanced the chemotactic activity of native human C5a des Arg but had no effect on the chemotactic activity of either porcine C5a or porcine C5a des Arg. Furthermore, porcine serum failed to enhance the chemotactic activity of native human C5a des Arg (Table IV), which indicated that, in contrast to human serum, porcine serum does not contain cochemotaxin-like activity. These results support our findings using human $\mathrm{C5a}$ des Arg-CHO (Fig. 1) and provide further evidence that the cochemotaxin interacts with the oligosaccharide portion of native human C5a des Arg.

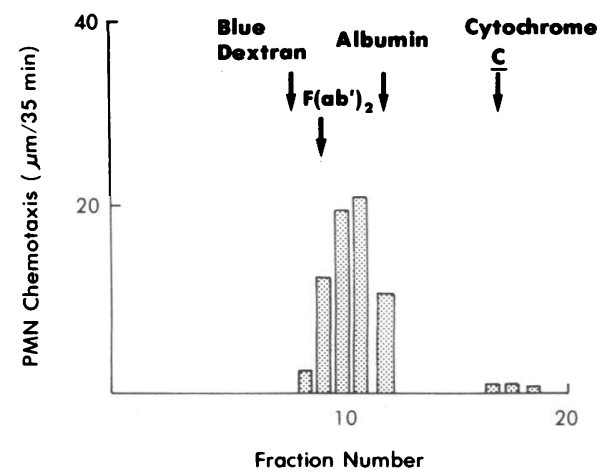

Figure 6. Elution profile of chemotactic activity after molecular sieve chromatography of ZTS on Bio-Gel P-150. Chromatography of ZTS $(0.2 \mathrm{ml})$ was performed on a $1.0 \times 20-\mathrm{cm}$ column of Bio-Gel P-150, and fractions $(0.4 \mathrm{ml})$ were tested at a concentration of $2.5 \%(\mathrm{vol} / \mathrm{vol})$ for chemotactic activity.
Table IV. Effects of Human Cochemotaxin, Porcine Serum, and Rabbit Serum on the Chemotactic Activities of Porcine C5a, Porcine C5a des Arg, and Human C5a des Arg

\begin{tabular}{lcc}
\hline & \multicolumn{2}{l}{ PMN migration* } \\
\cline { 2 - 3 } Stimulus & $\begin{array}{l}\text { No } \\
\text { cochemotaxin }\end{array}$ & $\begin{array}{l}10 \mathrm{ng} / \mathrm{ml} \\
\text { cochemotaxin }\end{array}$ \\
\hline & $\mu \mathrm{m} / 35 \mathrm{~min}$ & $\mu \mathrm{m} / 35 \mathrm{~min}$ \\
Buffer & $83.8 \pm 2.2$ & \\
Porcine C5a $(0.5 \mathrm{ng} / \mathrm{ml})$ & $97.3 \pm 2.8 \S$ & $96.3 \pm 3.1 \S$ \\
Porcine C5a des Arg $(5.0 \mathrm{ng} / \mathrm{ml})$ & $83.7 \pm 2.3$ & $83.9 \pm 3.2$ \\
$\quad(10 \mathrm{ng} / \mathrm{ml})$ & $90.7 \pm 3.2$ & $89.9 \pm 3.7$ \\
Native human C5a des Arg & & \\
$\quad(5.0 \mathrm{ng} / \mathrm{ml})$ & $84.3 \pm 1.8$ & $108.1 \pm 2.1^{11}$ \\
$\quad+$ porcine serum $(10 \mu \mathrm{l})$ & $84.4 \pm 1.9$ & \\
$\quad+$ rabbit serum $(10 \mu \mathrm{l})$ & $106.1 \pm 2.8^{\prime \prime}$ & \\
\end{tabular}

* Results represent mean values $( \pm \mathrm{SE})$ obtained in three experiments using PMN from different donors.

$\ddagger$ Neither porcine nor rabbit serum (heated at $56^{\circ} \mathrm{C}$ for $30 \mathrm{~min}$ ) exhibited chemokinetic activity.

$\S P$ vs. buffer alone $<0.025$ ( $t$ test).

" $P$ vs. buffer alone $<0.01$ ( $t$ test).

Finally, experiments were performed to determine whether rabbit serum was capable of enhancing the chemotactic activity of human C5a des Arg (i.e., contained cochemotaxin-like activity). Jose et al. (26) reported that human C5a and C5a des Arg were similar with respect to their ability to induce PMN-dependent inflammation when injected intradermally in rabbits. These authors prepared C5a des Arg by incubating human C5a with rabbit serum (as a source of carboxypeptidase $\mathrm{N}$ ). The mixture of C5a des Arg plus rabbit serum was then injected into rabbit skin and its ability to increase vascular permeability compared with that of purified human C5a. One possible explanation for these results was that rabbit serum augmented the biologic activity of native human C5a des Arg. Consequently, it was of interest to determine whether rabbit serum exhibited cochemotaxin-like activity. As Table IV shows, rabbit serum, at a concentration that by itself did not induce PMN-directed migration, was capable of enhancing the chemotactic activity of a suboptimal concentration of native human C5a des Arg.

\section{Discussion}

As Table I shows, highly purified C5a des Arg-CHO was significantly more potent than native C5a des Arg as a chemoattractant for human PMN. These observations are similar to those reported previously by Gerard et al. (11), and support the suggestion that the oligosaccharide chain acts as a negative modulator of the chemotactic activity of native C5a des Arg.

As we reported previously (5), the chemotactic activity of native C5a des Arg was enhanced significantly, and in a concentration-dependent fashion, by purified cochemotaxin (Fig. 1). The cochemotaxin, however, had no effects at all on the chemotactic activity exhibited by either suboptimal concentrations of $\mathrm{C} 5 \mathrm{a}$ des Arg-CHO (Fig. 1) or suboptimal concentrations of C5a and FMLP (not shown). The cochemotaxin also failed to enhance the chemotactic activity of porcine C5a des Arg (Table IV), a polypeptide devoid of carbohydrate (11). 
In light of these findings, we considered the possibility that the cochemotaxin interacts directly with the oligosaccharide chain of native C5a des Arg. Indeed, we already had some evidence (albeit indirect) that the cochemotaxin interacts with sugar moieties. We reported previously that the apparent molecular weight of the cochemotaxin, as determined by SDS PAGE $(60,000)$, differed substantially from that estimated by molecular sieve chromatography on Sephadex G-75 $(20-30,000)(4,5)$. Moreover, we demonstrated that the elution profile of cochemotaxin activity observed after Sephadex G-75 chromatography of heat-inactivated human serum was similar to the elution profile of low molecular weight, C5-derived chemotactic activity observed when zymosan-activated serum was chromatographed under identical conditions $(4,5)$. It seemed likely, therefore, that binding of the cochemotaxin to Sephadex (agarose) gels accounted for its retarded elution and, consequently, for its coelution with low molecular weight C5-derived peptides (predominantly C5a des Arg). Such binding was not observed with polyacrylamide gels. The purified cochemotaxin eluted from columns of Bio-Gel P-150 with an apparent molecular weight of 60,000 (5).

Evidence that the cochemotaxin interacts with the oligosaccharide chain of native C5a des Arg was obtained in several ways. First, of the known components of the oligosaccharide chain (13), only sialic acid was capable of preventing enhancement by the cochemotaxin of the chemotactic activity exhibited by suboptimal concentrations of native C5a des Arg (Table II). This effect of sialic acid was concentration-dependent (Fig. 2) and specific. Sialic acid did not influence PMN migration toward chemotactic concentrations of either native $\mathrm{C} 5 \mathrm{a}$, native $\mathrm{C5a}$ des Arg alone, or FMLP (Table III).

It is unlikely that the effect of sialic acid was due to its negative charge. The polyanion, heparin $(0.5-5.0 \mathrm{U} / \mathrm{ml})$, failed to influence the chemotactic activity exhibited by mixtures of native C5a des Arg and its cochemotaxin (Table III). Furthermore, both native and neuroaminidase-treated cochemotaxin were equipotent with respect to their ability to enhance the chemotactic activity of native C5a des Arg (Table III). Native cochemotaxin, however, had no effect on the chemotactic activity of neuraminidase-treated C5a des Arg. Interestingly, treatment of native C5a des Arg with neuraminidase enhanced its chemotactic activity for PMN. These findings suggested, therefore, that the cochemotaxin formed a physical complex with native C5a des Arg by attaching to a siliac acid residue on the oligosaccharide chain, rather than by an electrostatic interaction. Additional support for this suggestion came from studies using acid PAGE, molecular sieve chromatography, and centrifugation on sucrose density gradients.

Electrophoretic migration of radiolabeled C5a des Arg into acid polyacrylamide gels was retarded significantly by the cochemotaxin (Fig. 3). Interestingly, this effect of the cochemotaxin was reversed by adding $5.0 \mathrm{mM}$ sialic acid to the mixture before electrophoresis. It should be noted, however, that while these results suggested that the cochemotaxin interacts with native C5a des Arg, it could not be concluded that such an interaction leads to the formation of a physical complex between the two polypeptides. Evidence of this was obtained from experiments using molecular sieve chromatography and centrifugation on sucrose density gradients.

When examined by chromatography on polyacrylamide gels, ${ }^{125}$ I-C5a des Arg eluted with an apparent molecular weight of $\sim 11,000$ (Fig. $4 \mathrm{~A}$ ). Chromatography of ${ }^{125} \mathrm{I}-\mathrm{C} 5 \mathrm{a}$ des Arg plus unlabeled cochemotaxin, however, yielded a peak of radioactivity that eluted with an apparent molecular weight of 85,000. As in the experiments using PAGE, the effect of the cochemotaxin on the elution profile of radiolabeled C5a des Arg was prevented by sialic acid. To confirm these results, similar experiments were performed with ${ }^{125}$ I-labeled cochemotaxin and either unlabeled native C5a des Arg or unlabeled C5a des Arg-CHO. When radiolabeled cochemotaxin was chromatographed either alone or in combination with $\mathrm{C} 5 \mathrm{a}$ des $\mathrm{Arg}-\mathrm{CHO}$, all of the radioactivity eluted with an apparent molecular weight of 60,000 (Fig. 4 B). In contrast, when a mixture of ${ }^{125} \mathrm{I}$-cochemotaxin plus unlabeled native C5a des Arg was chromatographed under identical conditions, a single peak of radioactivity (containing chemotactic activity) eluted with an apparent molecular weight of 85,000. These results suggest further that the cochemotaxin does form a biologically active, physical complex with C5a des Arg, possibly by interacting with sialic acid residues on the oligosaccharide chain of the molecule. Electrostatic forces do not appear to play a major role in the formation of the complex, because ${ }^{125} \mathrm{I}-\mathrm{co}-$ chemotaxin plus C5a des Arg eluted with the same apparent molecular weight when chromatographed under conditions of low and high ionic strength. Further evidence that C5a des Arg and the cochemotaxin form a true physical complex was obtained using sucrose density gradient ultracentrifugation (Fig. 5). Mixtures of ${ }^{125} \mathrm{I}$-C5a des Arg and cochemotaxin exhibited an apparent molecular weight of $85,300 \pm 3,000$, while ${ }^{125}$ I-C 5 a des Arg alone

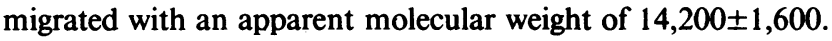
These results indicate that the cochemotaxin forms a physical complex with C5a des Arg, with a molar ratio of cochemotaxin to C5a des Arg of $\sim 1: 2$.

It should be emphasized that although attachment of the cochemotaxin to C5a des Arg appears to be saccharide specific, it may not be protein specific. Indeed, in preliminary experiments, high concentrations $(>100 \mathrm{ng} / \mathrm{ml}$ ) of the sialylated glycoprotein, orosomucoid, prevented enhancement by the cochemotaxin of the chemotactic activity exhibited by mixtures of suboptimal concentrations of C5a des Arg and the cochemotaxin (data not shown). It is possible, therefore, that the cochemotaxin attaches to a number of circulating and cell surface glycoproteins.

The data presented here may explain, in part, the finding reported by Beebe et al. (8) that zymosan-activated serum contains an anionic, C5-derived chemotactic factor with an apparent molecular weight of 65,000 . It is possible that this factor is a complex of one molecule of C5a des Arg plus its cochemotaxin. Further evidence that this might be the case was obtained in experiments in which we isolated C5-derived chemotactic activity after molecular sieve chromatography of ZTS on Bio-Gel P-150. The bulk of chemotactic activity, which was heat $\left(56^{\circ} \mathrm{C}\right.$ for 30 min)-stable and inhibited by antibodies to human $\mathrm{C} 5$, eluted with an apparent molecular weight of $80-85,000$ (Fig. 6). The elution profile of chemotactic activity observed after chromatography of ZTS on Bio-Gel P-150 was similar to the elution profile of chemotactic activity and radioactivity observed when ${ }^{125}$ I-cochemotaxin and $\mathrm{C} 5 \mathrm{a}$ des Arg were chromatographed under identical conditions (Fig. $4 \mathrm{~B}$ ). These data may also explain the finding reported by Jose et al. (26), that native human C5a des Arg (plus rabbit serum) resembled human C5a with respect to its ability to increase vascular permeability when injected into rabbit skin. It is likely that the biologic activity of human C5a des Arg was enhanced by the cochemotaxin-like activity present in rabbit serum. This finding suggests that the cochemotaxin may play a role in modulating inflammation in vivo. 
In summary, we confirmed that $\mathrm{C} 5 \mathrm{a}$ des Arg-CHO is significantly more potent than native $\mathrm{C} 5 \mathrm{a}$ des Arg as a chemoattractant for human PMN. In addition, we found that the cochemotaxin does not influence the chemotactic activity of C5a des Arg-CHO. Finally, we found that the cochemotaxin forms of physical complex with native C5a des Arg, possibly by attaching to a sialic acid residue on the oligosaccharide portion of the molecule. It is intriguing to speculate that the cochemotaxin enhances the chemotactic activity of native C5a des Arg by masking the oligosaccharide chain in such a fashion as to make the molecule resemble $\mathrm{C} 5 \mathrm{a}$ des Arg-CHO.

\section{Acknowledgments}

We thank Dr. Tony Hugli, Scripps Clinic and Research Foundation, La Jolla, CA, for providing the preparation of purified porcine $\mathrm{C} 5 \mathrm{a}$.

\section{References}

1. Perez, H. D. 1984. Complement-derived chemotactic factors and their relevance to disease. Crit. Rev. Oncol. Hematol. 1:1-19.

2. Fernandez, H. N., P. M. Henson, A. Otani, and T. Hugli. 1978. Chemotactic responses to human $\mathrm{C} 3 \mathrm{a}$ and $\mathrm{C} 5 \mathrm{a}$ anaphylatoxins. I. Evaluation of $\mathrm{C} 3 \mathrm{a}$ and $\mathrm{C} 5 \mathrm{a}$ leukotaxis in vitro and under simulated in vivo conditions. J. Immunol. 120:109-115.

3. Webster, R. O., S. R. Hong, R. B. Johnston, Jr., and P. M. Henson. 1980. The biological effects of the human complement fragments C5a and C5a des Arg on neutrophil function. Immunopharmacology. 2:201219.

4. Perez, H. D., I. M. Goldstein, D. Chernoff, R. O. Webster, and P. M. Henson. 1980. Chemotactic activity of C5a des Arg: Evidence of a requirement for an anionic "helper factor" and inhibition by a cationic protein in serum from patients with systemic lupus erythematosus. Mol. Immunol. 17:163-169.

5. Perez, H. D., I. M. Goldstein, R. O. Webster, and P. M. Henson. 1981. Enhancement of the chemotactic activity of human C5a des Arg by an anionic polypeptide ("cochemotaxin") in normal serum and plasma. J. Immunol. 126:800-804.

6. Hugli, T. E., and H. J. Müller-Eberhard. 1978. Anaphylatoxins: C3a and C5a. Adv. Immunol. 26:1-53.

7. Bokisch, V. A., and H. J. Müller-Eberhard. 1970. Anaphylatoxin inactivator of human plasma: its isolation and characterization as a carboxypeptidase. J. Clin. Invest. 49:2427-2436.

8. Beebe, D. P., P. A. Ward, and J. K. Spitznagel. 1980. Isolation and characterization of an acidic chemotactic factor from complementactivated human serum. Clin. Immunol. Immunopathol. 15:88-105.

9. Kreutzer, D. L., S. Kunkel, P. A. Ward, H. Showell, and R. H. McLean. 1980. Preservation of C3, C5, and C5a functional activities by a new radiolabelling method: demonstration of $\mathrm{C} 5$ products in complement-activated normal serum. J. Immunol. 123:2278-2282.
10. Gerard, C., and T. E. Hugli. 1981. Identification of classical anaphylatoxin as the des-Arg form of the C5a molecule: evidence for a modulator role for the oligosaccharide unit in human des Arg-74-C5a. Proc. Natl. Acad. Sci. USA. 78:1833-1837.

11. Gerard, C., D. E. Chenoweth, and T. E. Hugli. 1981. Response of human neutrophils to C5a: a role for the oligosaccharide moiety of human C5a des Arg-74 but not C5a in biologic activity. J. Immunol. 127:1978-1982.

12. Hugli, T. E., C. Gerard, M. Kawahara, M. E. Scheetz, II, R. Barton, S. Briggs, G. Koppel, and S. Russell. 1981. Isolation and characterization of three separate anaphylatoxins from complement-activated human serum. Mol. Cell. Biochem. 41:59-66.

13. Fernandez, H. N., and T. E. Hugli. 1979. Primary structural analysis of the polypeptide portion of human C5a anaphylatoxin. Polypeptide sequence determination and assignment of the oligosaccharide attachment site in C5a. J. Biol. Chem. 253:6955-6964.

14. Radioiodination of proteins with Enzymobeads. 1981. Technical Bulletin 1071. BioRad Laboratories, Richmond, CA.

15. Chenoweth, D. E., M. G. Goodman, and W. O. Weigle. 1982. Demonstration of a specific receptor for human C5a anaphylatoxin on murine macrophages. J. Exp. Med. 156:68-78.

16. Chenoweth, D. E., and T. E. Hugli. 1978. Demonstration of specific C5a receptor on intact human polymorphonuclear leukocytes. Proc. Natl. Acad. Sci. USA. 75:3943-3947.

17. Perez, H. D., R. R. Ong, and F. Elfman. 1985. Removal or oxidation of surface membrane sialic acid inhibits formyl-peptide-induced polymorphonuclear leukocyte chemotaxis. J. Immunol. 134:1902-1908.

18. Boyum, A. 1968. Isolation of mononuclear cells and granulocytes from human blood. Isolation of mononuclear cells by one centrifugation, and of granulocytes by combining centrifugation and sedimentation at 1 g. Scand. J. Clin. Lab. Invest. 21(Suppl. 97):77-87.

19. Perez, H. D., M. Lipton, and I. M. Goldstein. 1978. A specific inhibitor of complement (C5)-derived chemotactic activity in serum from patients with systemic lupus erythematosus. J. Clin. Invest. 62:29-38.

20. Zigmond, S. H., and J. G. Hirsch. 1973. Leukocyte locomotion and chemotaxis: new methods for evaluation and demonstration of a cell-derived chemotactic factor. J. Exp. Med. 137:387-410.

21. Keller, H. U., M. W. Hess, and H. Cottier. 1977. The chemokinetic effect of serum albumin. Experientia (Basel). 33:1386-1387.

22. Maderazo, E. G., and C. L. Woronick. 1978. Micropore filter assay of human granulocyte locomotion: problems and solutions. Clin. Immunol. Immunopathol. 11:196-211.

23. Martin, R. G., and B. N. Ames. 1961. A method for determining the sedimentation behaviour of enzymes: application to protein mixtures. J. Biol. Chem. 236:1372-1379.

24. Ward, P. A., and L. J. Newman. 1969. A neutrophil chemotactic factor from human C5. J. Immunol. 102:93-99.

25. Robinson, L. D., S. K. Wooten, and M. E. Miller. 1977. Partial characterization of in vivo chemotactic activity: comparison to human C5a. J. Allergy Clin. Immunol. 59:353-358.

26. Jose, P. J., M. J. Forrest, and T. J. Williams. 1981. Human C5a Arg increases vascular permeability. J. Immunol. 127:2376-2380. 